\title{
A Delphi consensus statement of the Neuropathic Pain Special Interest Group of the Italian Neurological Society on pharmacoresistant neuropathic pain
}

\author{
P. Ciaramitaro ${ }^{1}$ - G. Cruccu ${ }^{2}$ - M. de Tommaso ${ }^{3}$ - G. Devigili ${ }^{4}$ - D. Fornasari ${ }^{5} \cdot$ P. Geppetti ${ }^{6} \cdot$ M. Lacerenza $^{7}$. \\ G. Lauria ${ }^{8,9} \cdot$ S. Mameli ${ }^{10}$ - P. Marchettini ${ }^{6,11,12}$ - M. Nolano ${ }^{13,14}$ - E. Polati ${ }^{15}$ • V. Provitera ${ }^{16}$ - M. Romano ${ }^{17}$. \\ C. Solaro ${ }^{18} \cdot$ S. Tamburin ${ }^{19} \cdot$ V. Tugnoli $^{20} \cdot$ M. Valeriani $^{21,22} \cdot$ Andrea Truini $^{2}$ (I) $\cdot$ On behalf of the Neuropathic Pain \\ Special Interest Group of the Italian Neurological Society
}

Received: 18 December 2018 / Accepted: 23 March 2019 / Published online: 2 April 2019

(C) Fondazione Società Italiana di Neurologia 2019

\begin{abstract}
To improve patient care and help clinical research, the Neuropathic Pain Special Interest Group of the Italian Neurological Society appointed a task force to elaborate a consensus statement on pharmacoresistant neuropathic pain. The task force included 19 experts in neuropathic pain. These experts participated in a Delphi survey consisting of three consecutive rounds of questions and a face-to-face meeting, designed to achieve a consensus definition of pharmacoresistant neuropathic pain. In the three rounds of questions, the participants identified and described the main distinguishing features of pharmacoresistance. In the face-to-face meeting the participants discussed the clinical features determining pharmacoresistance. They finally agreed that neuropathic pain is pharmacoresistant when "the patient does not reach the $50 \%$ reduction of pain or an improvement of at least 2 points in the Patient Global Impression of Change, having used all drug classes indicated as first, second, or third line in the most recent and widely agreed international guidelines, for at least 1 month after titration to the highest tolerable dose." Our consensus statement might be useful for identifying eligible patients for invasive treatments, and selecting patients in pharmacological trials, thus improving patient care and helping clinical research.
\end{abstract}

Keywords Neuropathic pain $\cdot$ Pharmacoresistance $\cdot$ Painful neuropathy $\cdot$ Refractory pain

\section{Introduction}

Neuropathic pain-i.e., pain arising directly from a lesion or disease that affects the somatosensory nervous system - is a common clinical problem (http://www.iasp-pain.org/ Education/Content.aspx?ItemNumber=1698). Neuropathic pain, having a prevalence in the range of $7-10 \%$, represents a significant economic and social burden [1]. Despite the advances, a considerable number of patients do not get adequate pain relief or improvement in quality of life from available drugs. Meta-analysis [2] as well as experienced clinicians claim that neuropathic pain is pharmacoresistant in about $50 \%$ of patients [3].

Andrea Truini

andrea.truini@uniroma1.it

Extended author information available on the last page of the article
Although pharmacoresistant neuropathic pain is a common clinical problem [3,4], a precise definition of pharmacoresistance remains elusive. In most studies assessing the efficacy of invasive procedures for patients with pharmacoresistant neuropathic pain, the definition criterion of pharmacoresistance is frequently ambiguous $[5,6]$. The current guideline on the clinical development of medicinal products intended for the treatment of pain, issued by the European Medicine Agency (https://www.ema.europa.eu/ documents/scientific-guideline/draft-guideline-clinicaldevelopment-medicinal-products-intended-treatment-pain en-0.pdf), does not provide any indication on how to define pharmacoresistance to neuropathic pain. A pragmatic definition of pharmacoresistant neuropathic pain may be therefore useful for identifying patients for invasive treatments in clinical practice, or for selecting patients in pharmacological trials.

The aim of this study, issued by the Neuropathic Pain Special Interest Group (NeuPSIG) of the Italian 
Neurological Society, was to reach a consensus statement on how to define pharmacoresistant neuropathic pain. To do so a task force participated into three consecutive rounds of questionnaires, and in a face-to-face meeting designed to achieve a final consensus statement on pharmacoresistance definition.

\section{Methods}

The NeuPSIG of the Italian Neurological Society organized this Consensus, based on the Delphi technique, between April and June 2018. The task force included 15 neurologists, two pharmacologists, and two pain physicians, selected because of their specific expertise in neuropathic pain. The participants were selected due to their scientific activity in the neuropathic pain field, the geographical representation of the Italian territory, and the clinical and scientific activity associated with the Italian Neurological Society. For instance, several members of this team participated in relatively recent epidemiological studies dealing with neuropathic pain [7-9]. These experts were contacted by email with an invitation to participate in the Delphi method survey.

We organized three rounds of open questions, sent by email to the expert panel. Two facilitators (AT and GC) developed the questions. After each round, the two facilitators analyzed the responses and elaborated the successive round questions. Each participant was unaware of the responses of the other participants.

Of the 19 invited experts, 17 completed the three rounds and 16 participated to the face-to-face meeting. In this consensus procedure, we set a priori a level of agreement at $80 \%$.

The response rate for the three rounds of questions ranged between 82 and $100 \%$.

\section{Rounds of questions}

In the first round, we sought the general opinion of the participants about the problem of treatment-resistant neuropathic pain. We asked, "In your opinion when does neuropathic pain become "pharmacoresistant?"

For the second round, a summary of the results of round one was fed back to participants by email, along with questions of round 2. This round included items directly raised by the answers to the round 1 questions. Accordingly, the questions of the second round were focused to reach a precise definition of clinically meaningful pain relief, global impression of change, and how to identify effective drugs and their dosage and minimal duration of treatment, before defining a patient pharmacoresistant.

A summary of the results of round two was then fed back to participants by email. The third round aimed at completing and clarifying some of the issues raised by the replies to the second round. We therefore included questions on whether drugs of the same class (e.g., different tricyclic antidepressants) shall be tried, how comorbidities influence the response to treatment, and whether other, non-invasive, therapies (e.g., nutritional supplements, relaxation) shall be tried before defining a patient pharmacoresistant

\section{Face-to-face meeting}

After the three rounds of questions, we fed back a list of all the answers in anonymized form to participants. This procedure step was meant to prepare participants to the face-to-face meeting. In this meeting, the participants were asked to discuss the different items raised by the rounds of questions and indicate their agreement with each of the components to be incorporated into a working definition of pharmacoresistant neuropathic pain.

In the face-to-face meeting the participants also discussed about the possible differences between the terms pharmacoresistance and refractoriness, and sought an agreement on the distinguishing features of these two terms.

\section{Results}

\section{Rounds of questions}

The answers to the first question showed that all participants, though using different wording, indicated that neuropathic pain becomes pharmacoresistant when drugs do not provide sufficient pain relief or a sufficient global impression of change (Table 1).

In the second round, the participants indicated the definition of clinically meaningful pain relief as a reduction of pain of 30 or $50 \%$, as assessed with a validated pain scale (i.e., the Visual Analogue Scale and the Likert scale). Most participants indicated as a clinically meaningful global impression of change, a 2-point improvement (corresponding to "very much improved" or "much improved") in the Patient Global Impression of Change scale (PGIC), a 7-point scale commonly used in the management of patients with neuropathic pain (Fig. 1). Most participants considered as effective drugs those having at least a weak recommendation in international guidelines or recommendations. Virtually all the participants considered the adequate drug dosage as the best balance between effectiveness and tolerability; conversely, they provided widely varying definitions for adequate duration of drug trial, indicating a trial duration between 2 weeks and 6 months.

In the round 3 , according to several participants although no specific evidence is available, different drugs of the same classes should be tried, mainly due to possible differences in pharmacokinetic profiles. Concerning the influence of comorbidities, the participants indicated three main issues: 
Table 1 Round questions and exemplary responses

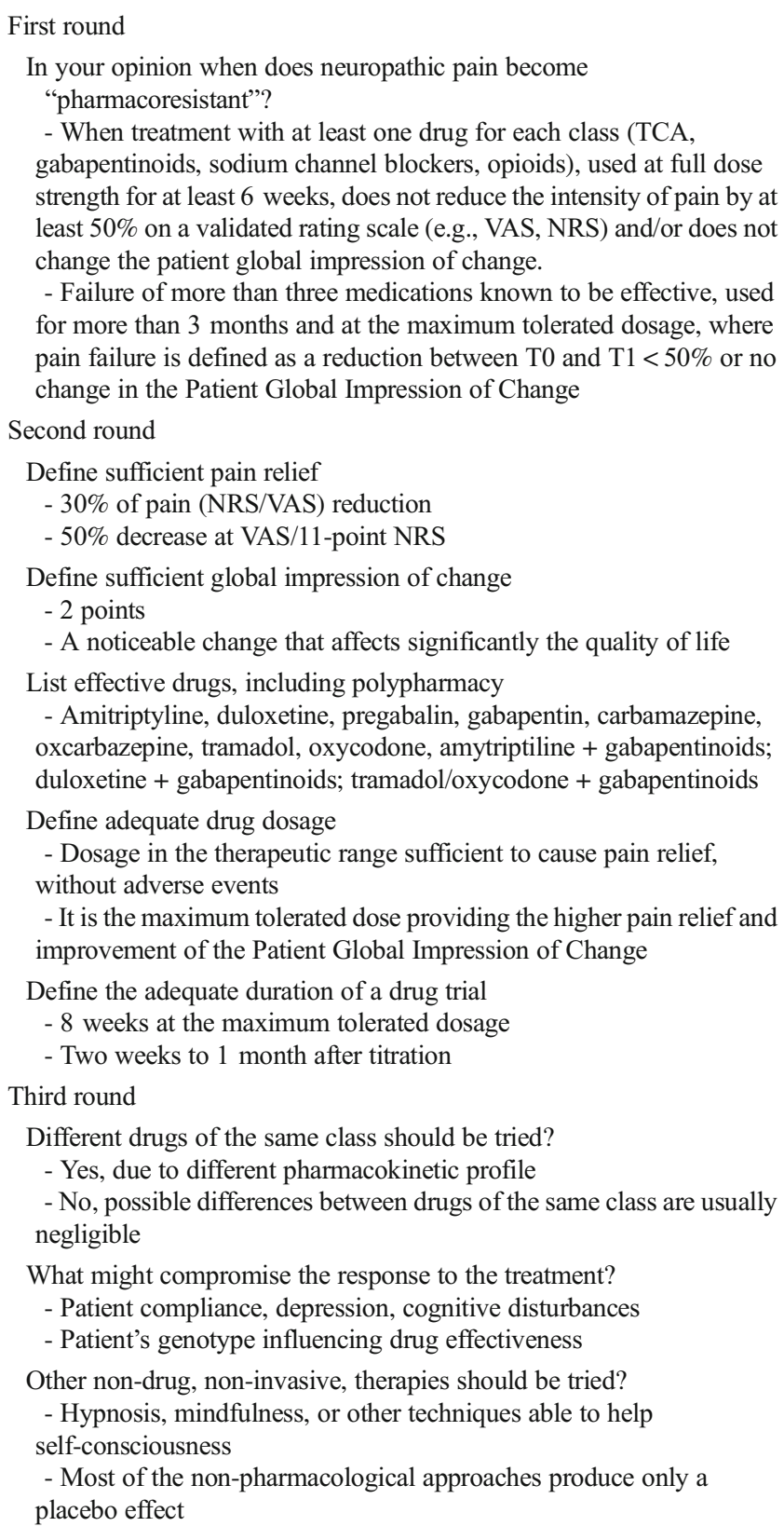

the patient compliance and adherence to therapy, psychiatric comorbidities, and patient's genotype influencing the drug effectiveness. Replies about additional treatments varied considerably among the participants. Some participants indicated among possible useful treatments cognitive and behavioral therapy, physiotherapy, acupuncture, relaxation, and nutritional supplements. However, many participants considered that the current evidence supporting the clinical use of these treatments is still insufficient (Fig. 1).

\section{Face-to-face meeting}

In the face-to-face meeting there was unanimous consensus that among the different issues raised during the three rounds of questions, pharmacoresistance definition should include the following items: satisfactory pain relief or PGIC, number of efficacious drugs, dosage of the drugs, and treatment duration. All participants agreed that satisfactory pain relief corresponds to the $50 \%$ reduction of pain and a clinically meaningful global impression of change corresponds to an improvement of at least 2 points in the PGIC. All the drugs indicated as first, second, or third line in the most recent international guidelines should be tried, for at least 1 month after titration, using the highest dose according to the EU approved Summary of Product Characteristics (SPC), without clinically important side effects.

All the participants agreed that pharmacoresistant and refractory neuropathic pain are distinct conditions. The definition of pharmacoresistance should be used only regarding pharmacological treatment; conversely, refractoriness is more relevant to the lack of response to any treatment, including neurostimulation and surgical procedures.

Box 1 Definition of pharmacoresistant neuropathic pain

Neuropathic pain is pharmacoresistant when "the patient does not reach the $50 \%$ reduction of pain or an improvement of at least 2 points in the Patient Global Impression of Change, having used all drug classes indicated as first, second, or third line in the most recent and widely agreed international guidelines, for at least 1 month after titration to the highest tolerable dose."

\section{Discussion}

In this study, using the Delphi method, a task force appointed by the NeuPSIG of the Italian Neurological Society, agreed that neuropathic pain is pharmacoresistant when "the patient does not reach the $50 \%$ reduction of pain or an improvement of at least 2 points in the PGIC, having used all drug classes indicated as first, second, or third line in the most recent and widely agreed international guidelines, for at least 1 month after titration to the highest tolerable dose." We believe that this consensus statement on pharmacoresistant neuropathic pain may serve as a pragmatic and applicable definition for the everyday clinical management and pharmacological trials.

The primary target users of our consensus definition of pharmacoresistant neuropathic pain are medical practitioners at all health care levels involved in the clinical management of patients with neuropathic pain. Our definition may help physicians, health care administrators, and insurers in identifying eligible patients for invasive therapeutic procedures. Other target users are clinical researchers because adopting a consensus definition might help for selecting patients in pharmacological trials. 


\section{Second Round}
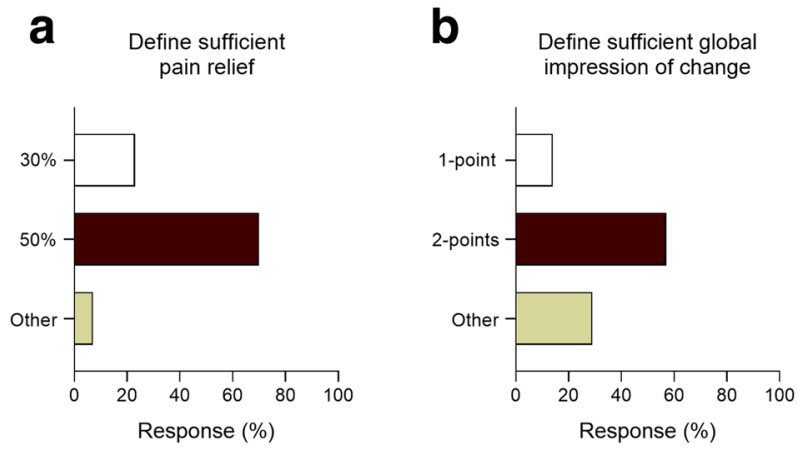

\section{Third Round}
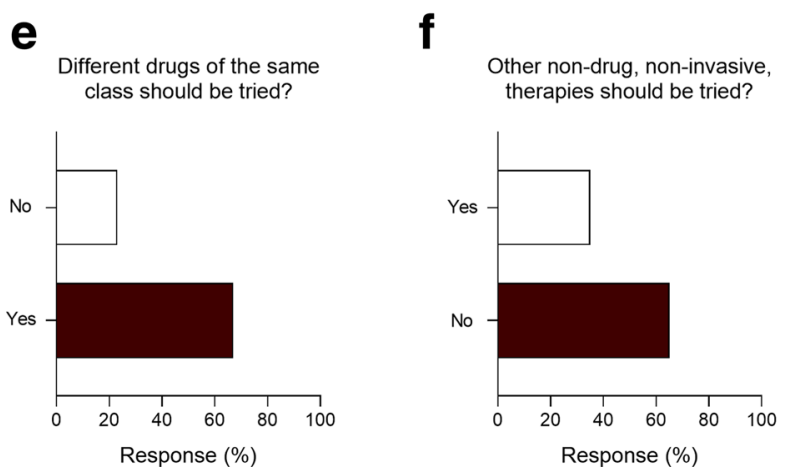

Fig. 1 Frequency of the different responses to round questions 2 and 3. Given that rounds 2 and 3 consisted of open questions, for some questions the two facilitators performed a qualitative analysis and grouped together similar items. For instance in the question D "Define the adequate

\section{The definition of satisfactory pain relief and PGIC}

How to define satisfactory pain relief remains a controversial problem. Many pharmacological trials assessing the efficacy of drugs in patients with neuropathic pain indicate as primary endpoint the $30 \%$ reduction of pain [2]. However, several studies showed that clinically important improvement of quality of life and health status are associated with pain relief higher than the $30 \%$ reduction of pain $[10,11]$. Furthermore, the percent change that is clinically important to patient increases as the baseline pain severity increases. Hence, for patients with severe pain, the percentage of pain relief should be higher than $30 \%$. For these reasons, we decided to indicate the $50 \%$ reduction of pain as the appropriate target for pharmacological treatment, given that a pain reduction of $\geq 50 \%$ probably represents the substantial change in pain severity for most patients [12].

In the definition of pharmacoresistant pain we decided to include also the PGIC. The percent change of pain and the PGIC are closely related; this correlation, however, loses consistency in patients with high pain scores. Furthermore many
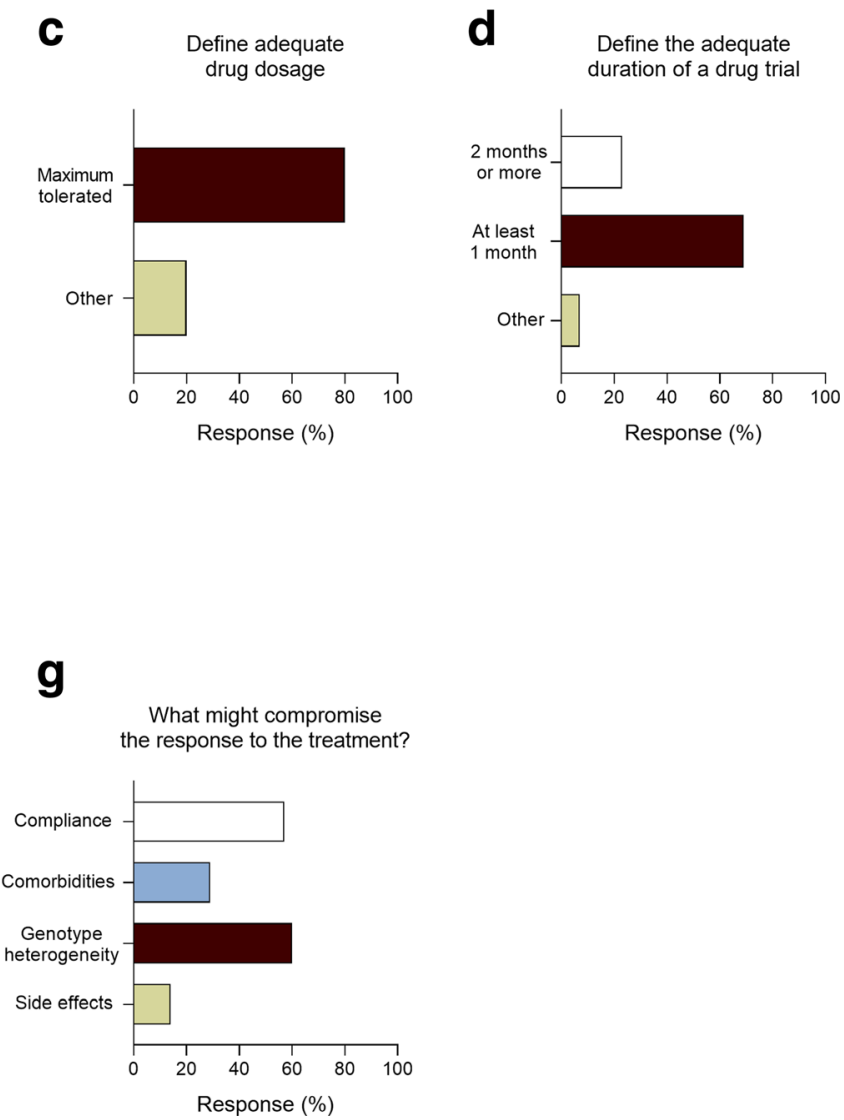

duration of a drug trial," the answers reporting a time span between 8 weeks and 6 months have been included in the item "2 months or more." The sum of percentage of answers to question $\mathrm{G}$ is above $100 \%$ given that some participants provided more than one answers

drugs used for neuropathic pain treatment (e.g., antidepressants and gabapentinoids) may improve sleep, mood, and therefore quality of life, regardless of the degree of pain reduction [13]. We therefore believe that the PGIC is a useful, alternative item to pain reduction. It follows that we may consider drug responders those patients with a pain relief lower than 50\%, but with an adequate improvement of PGIC. We identified as an adequate improvement of the PGCI a change of 2 points corresponding to "very much improved" or "much improved," given that this improvement notoriously corresponds to a clinically important change in the general health status [12].

\section{The definition of effective pharmacological treatment}

Many drugs are supposed to modulate somatosensory nervous system activity, thus possibly reducing pain. Only some drugs, however, have been tested in randomized controlled trials proving some efficacy in reducing neuropathic pain. Accordingly, in the definition of pharmacoresistance we referred to all the drug classes indicated as first, second, or third 
line in the most recent, widely agreed international guidelines based on meta-analysis of published randomized controlled trials. We propose that the most recent and appropriate guidelines are used. Currently, the guideline for neuropathic pain treatment issued by the Neuropathic Pain Special Interest Group of the International Association for the Study of Pain [2] should be considered as the reference guideline unless an updated guideline is published.

In the definition of pharmacoresistance we indicated that the minimal duration of treatment necessary to consider the drug insufficient should be of at least 1 month after titration. This choice reflects two main considerations. Drugs used for treating neuropathic pain are usually carefully titrated for minimizing adverse events, and the adequate dosage is reached after several days or weeks. While the analgesic effects of antidepressants on pain manifest in a few days to 1 week, the effects on depression and quality of life take approximately 2 to 4 weeks [14], thus possibly having late influence on the PGIC.

The magnitude of pain relief usually correlates with the drug dosage: the higher the dosage is, the larger the pain relief is $[15,16]$. Admittedly, this relationship has not been homogenously reported for all drugs. For instance whereas the efficacy of pregabalin linearly increases with the dosage, the efficacy of 60 and $120 \mathrm{mg}$ of duloxetine does not differ $[17,18]$. Regardless of the dose-response relationship, however, the frequency and severity of adverse events increases with the drug dosage [19]. We have therefore indicated that the drug dosage should aim at the highest dosage, according to the EU approved Summary of Product Characteristics, without clinically important side effects (i.e., the highest tolerable dose).

\section{Pharmacoresistance and refractoriness}

Although most clinical trials assessing drug efficacy have used pharmacoresistance and refractoriness as synonyms [20-22], some studies suggested that the two definitions might be perceived as different entities $[3,4]$. We suggest that while the term pharmacoresistance explicitly refers to drugs, the term refractoriness should be intended for neuropathic pain resistant also to non-pharmacological treatment (e.g., neurostimulation, physical therapies, cognitive-behavioral therapy). We believe that a clear-cut distinction between the two terms might be clinically useful, given that the pharmacoresistance is a requisite for patients' eligibility to invasive procedures.

\section{Limitations of the study}

Our expert panel mainly includes neurologists, given that the study is issued by the NeuPSIG of the Italian Neurological Society. This unbalanced team might be regarded as a limitation, because different medical practitioners may be involved in the clinical management of patients with neuropathic pain. However, neuropathic pain is due to neurological diseases. We therefore believe that the special role for neurologists in this field is largely expected and justified.

In this study using the Delphi procedure we provide an expert opinion consensus, unsupported by evidence-based data. However, we believe that this limitation is intrinsic to the problem of pharmacoresistant neuropathic pain, given that no study has systematically addressed the pharmacoresistance and most trials including patients with pharmacoresistant neuropathic pain did not explicitly define the criteria for identifying patients with poor or absent response to treatment.

Although our definition of pharmacoresistance has some resembling points with two earlier expert opinion-based studies [3, 4], important differences still exist. In our study we explicitly provide previously unreported details on the pain relief, PGIC, and drugs, useful to yield a working definition of pharmacoresistance, essential in clinical practice. Particularly we identify the $50 \%$ reduction of pain rather than the $30 \%$, reported in a previous study [4], and precisely indicate the level of satisfactory PGIC. We also clearly indicate that the effective drugs should be identified based on standard reference guidelines. Differently from the two previously published expert opinion-based studies [3, 4], we also addressed the possible differences between pharmacoresistant and refractory neuropathic pain.

Although many participants considered that different drugs of the same class should be tried, we did not include this point in the pharmacoresistance definition. Admittedly, drugs of the same class might have a different effect for minor differences in pharmacodynamic profile or different pharmacokinetic properties. For instance among the tricyclic antidepressants, amitriptyline and imipramine have a stronger activity on voltage-gated sodium channels than nortriptyline and desipramine $[23,24]$. Gabapentin and pregabalin have minor pharmacodynamic differences (pregabalin has greater binding affinity for the alpha-2/delta- 1 subunit than gabapentin), and major differences in terms of pharmacokinetics, especially absorption (the intestinal absorption of pregabalin is not saturable) $[25,26]$. We considered, however, that the possible differences between drugs of the same class are usually negligible; furthermore, screening different drugs of the same class may require a long time, thus making a definition of pharmacoresistance unsuitable to clinical practice.

\section{Conclusions}

In this study, using a Delphi procedure to reach a consensus statement, we provide a working definition for pharmacoresistant neuropathic pain, possibly useful for identifying patients for invasive treatments in the everyday clinical 
practice, or including patients in pharmacological studies. Admittedly, our definition of pharmacoresistant neuropathic pain requires to be validated in a dedicated clinical trial, assessing the interobserver and intraobserver consistency in the clinical setting [27].

Acknowledgements Giorgio Cruccu received received honoraria as speaker, member of advisory boards, or consultant from Alfasigma, Angelini, Biogen, and Lilly.

Pierangelo Geppetti received honoraria for consultancy or speaking at symposia and research financial support from Amgen Allergan, Chiesi, Elettrocore, IBSA, Menarini, Novartis, Pfizer, Sanofi, and TEVA.

Marina de Tommaso received honoraria for speaking at symposia or research financial supports from Allergan, Novartis, and Mundipharma.

Diego Fornasari has received honoraria as speaker, member of advisory boards, or consultant from Angelini, Alfasigma, Abiogen, Grunenthal, Pfeizer, and SPA.

Paolo Marchettini received honoraria for speaking at symposia and training the field force from Angelini, Chiesi, Epitech, Eli Lilly, and Pfizer.

Enrico Polati received honoraria for speaking at symposia from Grunenthal.

Claudio Solaro served as advisory board the following companies: Biogen Idec, Merck Serono, Almirall, and GW Pharma. He received speaking honoraria from Biogen Idec, Merck Serono. He received research grants and support by the FISM (Fondazione Italiana Sclerosi Multipla).

Stefano Tamburin received honoraria for speaking at symposia or research financial support from Epitech, FB Health, Mundipharma, and Pfizer.

Andrea Truini received honoraria for speaking at symposia or research financial supports from Alpha-Sigma, Angelini, Epitech, FB Health, and Pfizer.

The other authors reported no conflict of interest to declare.

The face-to-face meeting was held during the congress of the Italian Society of Clinical Neurophysiology, receiving an unconditioned support by Pfizer.

\section{References}

1. Bouhassira D, Lantéri-Minet M, Attal N, Laurent B, Touboul C (2008) Prevalence of chronic pain with neuropathic characteristics in the general population. Pain 136(3):380-387

2. Finnerup NB, Attal N, Haroutounian S, McNicol E, Baron R, Dworkin RH, Gilron I, Haanpää M, Hansson P, Jensen TS, Kamerman PR, Lund K, Moore A, Raja SN, Rice AS, Rowbotham M, Sena E, Siddall P, Smith BH, Wallace M (2015) Pharmacotherapy for neuropathic pain in adults: a systematic review and meta-analysis. Lancet Neurol 14(2):162-173

3. Hansson PT, Attal N, Baron R, Cruccu G (2009) Toward a definition of pharmacoresistant neuropathic pain. Eur J Pain 13(5):439_ 440

4. Smith BH, Torrance N, Ferguson JA, Bennett MI, Serpell MG, Dunn KM (2012) Towards a definition of refractory neuropathic pain for epidemiological research. An international Delphi survey of experts. BMC Neurol 12:29-34

5. Rasche D, Rinaldi PC, Young RF, Tronnier VM (2006) Deep brain stimulation for the treatment of various chronic pain syndromes. Neurosurg Focus 21(6):E8

6. André-Obadia N, Mertens P, Lelekov-Boissard T, Afif A, Magnin M, Garcia-Larrea L (2014) Is life better after motor cortex stimulation for pain control? Results at long-term and their prediction by preoperative rTMS. Pain Physician 17(1):53-62

7. Ciaramitaro P, Padua L, Devigili G, Rota E, Tamburin S, Eleopra R, Cruccu G, Truini A (2017) Neuropathic pain special interest group of the Italian neurological society. Prevalence of neuropathic pain in patients with traumatic brachial plexus injury: a multicenter prospective hospital-based study. Pain Med 18(12):2428-2432

8. Solaro C, Cella M, Signori A, Martinelli V, Radaelli M, Centonze D, Sica F, Grasso MG, Clemenzi A, Bonavita S, Esposito S, Patti F, D'Amico E, Cruccu G, Truini A (2018) Neuropathic Pain Special Interest Group of the Italian Neurological Society. Identifying neuropathic pain in patients with multiple sclerosis: a cross-sectional multicenter study using highly specific criteria. J Neurol 265(4): $828-835$

9. Truini A, Spallone V, Morganti R, Tamburin S, Zanette G, Schenone A, De Michelis C, Tugnoli V, Simioni V, Manganelli F, Dubbioso R, Lauria G, Lombardi R, Jann S, De Toni Franceschini L, Tesfaye S, Fiorelli M, Spagnoli A, Cruccu G (2018) A crosssectional study investigating frequency and features of definitely diagnosed diabetic painful polyneuropathy. Pain. 159(12):26582666

10. Cepeda MS, Africano JM, Polo R, Alcala R, Carr DB (2003) What decline in pain intensity is meaningful to patients with acute pain? Pain 105(1-2):151-157

11. Farrar JT, Pritchett YL, Robinson M, Prakash A, Chappell A (2010) The clinical importance of changes in the 0 to 10 numeric rating scale for worst, least, and average pain intensity: analyses of data from clinical trials of duloxetine in pain disorders. J Pain 11(2):109 118

12. Dworkin RH, Turk DC, McDermott MP, Peirce-Sandner S, Burke LB, Cowan P, Farrar JT, Hertz S, Raja SN, Rappaport BA, Rauschkolb C, Sampaio C (2009) Interpreting the clinical importance of group differences in chronic pain clinical trials: IMMPACT recommendations. Pain 146(3):238-244

13. Turk DC, Dworkin RH, Allen RR, Bellamy N, Brandenburg N, Carr DB, Cleeland C, Dionne R, Farrar JT, Galer BS, Hewitt DJ, Jadad AR, Katz NP, Kramer LD, Manning DC, McCormick CG, McDermott MP, McGrath P, Quessy S, Rappaport BA, Robinson JP, Royal MA, Simon L, Stauffer JW, Stein W, Tollett J, Witter J (2003) Core outcome domains for chronic pain clinical trials: IMMPACT recommendations. Pain 106:337-345

14. Fishbain DA, Detke MJ, Wernicke J, Chappell AS, Kajdasz DK (2008) The relationship between antidepressant and analgesic responses: findings from six placebo-controlled trials assessing the efficacy of duloxetine in patients with major depressive disorder. Curr Med Res Opin 24(11):3105-3015

15. Boyle J, Eriksson ME, Gribble L, Gouni R, Johnsen S, Coppini DV, Kerr D (2012) Randomized, placebo-controlled comparison of amitriptyline, duloxetine, and pregabalin in patients with chronic diabetic peripheral neuropathic pain: impact on pain, polysomnographic sleep, daytime functioning, and quality of life. Diabetes Care 35(12):2451-2458

16. Juhn MS, Parsons B, Varvara R, Sadosky A (2015) Pregabalin for painful diabetic peripheral neuropathy: strategies for dosing, monotherapy vs. combination therapy, treatment-refractory patients, and adverse events. Curr Med Res Opin 31(5):1017-1026

17. Kajdasz DK, Iyengar S, Desaiah D, Backonja MM, Farrar JT, Fishbain DA, Jensen TS, Rowbotham MC, Sang CN, Ziegler D, McQuay HJ (2007) Duloxetine for the management of diabetic peripheral neuropathic pain: evidence-based findings from post hoc analysis of three multicenter, randomized, double-blind, placebo-controlled, parallel-group studies. Clin Ther 29(Suppl):25362544

18. Lunn MP, Hughes RA, Wiffen PJ (2014) Duloxetine for treating painful neuropathy, chronic pain or fibromyalgia. Cochrane Database Syst Rev (1):CD007115 
19. Zaccara G, Gangemi P, Perucca P, Specchio L (2011) The adverse event profile of pregabalin: a systematic review and meta-analysis of randomized controlled trials. Epilepsia 52(4):826-836

20. Attal N, Brasseur L, Guirimand D, Clermond-Gnamien S, Atlami S, Bouhassira D (2008) Are oral cannabinoids safe and effective in refractory neuropathic pain? Eur J Pain 8(2):173-177

21. Jann S, Francia A, Fruguglietti ME, De Toni Franceschini L, Sterzi R (2012) Efficacy and safety of intravenous immunoglobulin as adjuvant treatment for refractory neuropathic pain. Results of an open-label, multicenter study. Pain Med 13(10):1334-1441

22. Navarro A, Saldaña MT, Pérez C, Masramón X, Rejas J (2012) Costs and health resources utilization following switching to pregabalin in individuals with gabapentin-refractory neuropathic pain: a post hoc analysis. Pain Pract 12(5):382-393

23. Chen YW, Huang KL, Liu SY, Tzeng JI, Chu KS, Lin MT, Wang JJ (2004) Intrathecal tri-cyclic antidepressants produce spinal anesthesia. Pain 112(1-2):106-112
24. Kremer M, Salvat E, Muller A, Yalcin I, Barrot M (2016) Antidepressants and gabapentinoids in neuropathic pain: mechanistic insights. Neuroscience 338:183-206

25. Fornasari D (2017) Pharmacotherapy for neuropathic pain: a review. Pain Ther 6(Suppl 1):25-33

26. Cruccu G (2007) Treatment of painful neuropathy. Curr Opin Neurol 20(5):531-535

27. Téllez-Zenteno JF, Hernández-Ronquillo L, Buckley S, Zahagun R, Rizvi S (2014) A validation of the new definition of drug-resistant epilepsy by the International League Against Epilepsy. Epilepsia 55(6):829-834

Publisher's note Springer Nature remains neutral with regard to jurisdictional claims in published maps and institutional affiliations.

\section{Affiliations}

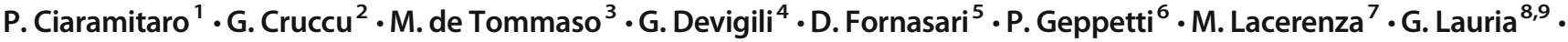
S. Mameli ${ }^{10} \cdot$ P. Marchettini ${ }^{6,11,12} \cdot$ M. Nolano ${ }^{13,14} \cdot$ E. Polati $^{15} \cdot$ V. Provitera ${ }^{16} \cdot$ M. Romano $^{17} \cdot$ C. Solaro ${ }^{18}$. S. Tamburin ${ }^{19} \cdot$ V. $^{\text {Tugnoli }}{ }^{20} \cdot$ M. Valeriani $^{21,22} \cdot$ Andrea Truini $^{2}$ (I)

1 Clinical Neurophysiology, Neuroscience Department, AOU Città della Salute e della Scienza di Torino, Torino, Italy

2 Department of Human Neuroscience, University Sapienza, Viale Università 30, 00185 Rome, Italy

3 Applied Neurophysiology and Pain Unit, Basic Medical Science, Neuroscience and Sensory System-SMBNOS-Department, Aldo Moro University, Bari, Italy

4 Neurological Unit 1, Fondazione I.R.C.C.S. Istituto Neurologico Carlo Besta, 20133 Milan, Italy

5 Department of Medical Biotechnology and Translational Medicine, Università degli Studi di Milano, Milan, Italy

6 Department of Health Sciences, Section of Clinical Pharmacology and Headache Center, University of Florence, Florence, Italy

7 Neurology and Pain Medicine Center, Humanitas, San Pio X Clinic, Milan, Italy

8 Neuroalgology Unit, IRCCS Foundation, "Carlo Besta" Neurological Institute, Milan, Italy

9 Department of Biomedical and Clinical Sciences "Luigi Sacco", University of Milan, Milan, Italy

10 Pain Therapy Unit, “A. Businco” Hospital, ASL 8, 09134 Cagliari, Italy

11 Pain Medicine Centre, Ospedale San Raffaele, Milan, Italy
12 University of Applied Science of Southern Switzerland, Pain Pathophysiology and Therapy Programme, Manno, Switzerland

13 Neurology Department, Skin Biopsy Laboratory, Istituti Clinici Scientifici Maugeri IRCCS, Via Maugeri, 4, 27100 Pavia, Italy

14 Department of Neurosciences, Reproductive Sciences and Odontostomatology, University Federico II of Naples, Naples, Italy

15 Department of Surgery, Dentistry, Maternal and Infant Sciences, Pain Therapy Centre, Verona University Hospital, Verona, Italy

16 Istituti Clinici Scientifici Maugeri IRCCS, Institute of Telese Terme (BN), Telese Terme, Italy

17 Neurology Unit, Azienda Ospedaliera Ospedali Riuniti Villa Sofia Cervello, Palermo, Italy

18 CRRF Mons L Novarese Moncrivello (VC), Moncrivello, Italy

19 Department of Neurosciences, Biomedicine and Movement Sciences, University of Verona, Verona, Italy

20 Neurology Unit, Azienda Ospedaliero-Universitaria, Ferrara, Italy

21 Division of Neurology, Ospedale Pediatrico Bambino Gesú, IRCCS, Rome, Italy

22 Center for Sensory-Motor Interaction, Aalborg University, Aalborg, Denmark 